\title{
Radiosurgery with Chemotherapy as an Alternative to RT for Glioblastoma Multiforme Patients 65 Years Old or Older: A Prospective Review of $\mathbf{4 0}$ Patients
}

Pietro Bortoletto ${ }^{1}$, Aizik L. Wolf ${ }^{2}$, Ariel Wolf ${ }^{3}$, Sammie Coy ${ }^{4}$, Laurie Blach ${ }^{5}$, Beatriz E. Amendola ${ }^{6}$, Lawrence Negret ${ }^{7}$, Francis Valdez-Albini ${ }^{7}$

1. Feinberg School of Medicine 2. Miami Neuroscience Center, Larkin Community Hospital 3. University of Chicago, Chicago, Illinois, The University of Chicago Medicine 4. Miami Neuroscience Center, Doctors Hospital, Coral Gables, Florida 5. Miami Neuroscience Center, Mount Sinai Medical Center, Miami Beach, Florida 6. Innovative Cancer Institute 7. Sylvester Comprehensive Cancer Center, University of Miami, Miami, Florida

$\square$ Corresponding author: Pietro Bortoletto, p.bortoletto@umiami.edu Disclosures can be found in Additional Information at the end of the article

\section{Abstract}

Introduction: Elderly patients have historically been treated with conventional external beam radiation therapy (EBRT) for glioblastoma multiforme (GBM).

Methods: We reviewed the results of treatment approaches, which included surgery, chemotherapy, and Gamma Knife (GK) radiosurgery, as an alternative to EBRT in this cohort of patients. Patients were treated during the period of 1999-2010 and were 65 years of age and older with histologically confirmed GBM.

Results: Forty patients, 65 years of age and older without previous radiation therapy (RT), were identified. Median age was 75 years (range 65-95). Median overall survival (OS) time was 10.9 months. Analysis showed that patients treated with GK, surgical resection, and chemotherapy (median OS of 14.2) had a significantly higher $(\mathrm{P}=.03)$ OS than patients treated with $\mathrm{GK}$, chemotherapy, and no surgical resection (median OS of 8.9 months) and than patients with GK, surgical resection, and no chemotherapy (median OS of 5.37 months).

Conclusions: In this study of glioblastoma patients over the age of 65 years with no previous RT, aggressive treatment with Gamma Knife radiosurgery, chemotherapy, and surgery is associated with improved OS.

Published 09/20/2011

\section{๑) Copyright 2011}

Bortoletto et al. This is an open access article distributed under the terms of the Creative Commons Attribution License CC-BY 3.0., which permits unrestricted use, distribution, and reproduction in any medium, provided the original author and source are credited.
Categories: Radiation Oncology, Neurosurgery

Keywords: radiosurgery, glioblastoma, elderly, gamma knife

\section{Introduction}

Glioblastoma multiforme (GBM) is the most common histological type of primary brain tumor in adults. It accounts for more than half of all adult primary brain tumors diagnosed annually in the United States [1]. GBM is most prevalent in patients 65-84 years of age, although this is the group that has least been studied in the literature [2]. An increase in the diagnosis of GBM's has been noted across the country. It is believed that stereotactic biopsy and the improvement and increased usage of imaging techniques have contributed to this upwards trend [3]. For those 
diagnosed, the current standard of care is surgical resection along with radiotherapy postoperatively [4]. The optimal management of this CNS neoplasm in the elderly is still a matter of debate, with several combinations beings studied. A number of small, prospective studies have shown the benefit of a tri-modal approach involving chemotherapy, radiation therapy, and surgical resection in elderly patients [4-8]. Most recently, RTOG studied the use of upfront stereotactic radiosurgery, radiation therapy, and carmustine $(\mathrm{BCNU})$ versus radiation therapy and BCNU alone. The results did not demonstrate additional difference in survival [9]. We are unaware of a study that studied radiosurgery alone with chemotherapy. We are interested in determining if our experience using Gamma Knife, chemotherapy, and surgery to treat GBM in patients 65 years old and older is comparable to the current standard of care with overall survival as the primary endpoint of this prospective study.

\section{Materials And Methods}

\section{Patients}

We prospectively treated 167 patients at least 65 years old with a clinical diagnosis of glioblastoma, all of whom had been treated solely at the Miami Neuroscience Center during the period of December 1993 to September 2010. Patient selection was based on the knowledge that patients had not undergone whole brain radiation or fractionated radiotherapy. Out of this group, we excluded 127 patients whom did not meet the aforementioned criteria or where pathology was not confirmed or available. This left 40 patients, 65 years of age and older with a histologically proven diagnosis of GBM and no previous radiation therapy. No other inclusion or exclusion criteria were applied.

\section{Treatments}

All patients had a pathologically confirmed diagnosis of GBM. Date of diagnosis was defined as the date of biopsy or surgery. We defined all surgical interventions beyond biopsy as "surgery", except as otherwise stated. We did not differentiate between a total or subtotal resection. The model Gamma Knife used changed throughout the course of the study, first beginning with the Model U, followed by the Model C, and currently, the Perfexion.

\section{Statistical analysis}

We defined overall survival (OS) time as the primary endpoint of this retrospective study. OS was measured from the date of diagnosis of glioblastoma multiforme. We performed uni- and multi-variate analyses to determine the differences in OS between our treatment modalities and our patients' KPS scores pre-treatment. We set the P value as .05 as the criterion for significance.

All tests of statistical significance were two-sided and all analyses were performed using Microsoft Excel for Mac 2011 (Microsoft Inc.).

\section{Results}

A full listing of patient and treatment characteristics can be found in Table 1 . The mean age was 73.1 years (SD 6.9 years; range, 65-95), and the gender distribution was slightly skewed in favor of females (55\% vs $45 \%$ ). All patients received Gamma Knife radiosurgery in a single treatment; some had multiple treatments to treat tumor recurrence or new lesions. The medial total dose was $11 \mathrm{~Gy}$ with a median of 9 isocenters to the $45 \%$ line. Chemotherapeutic agents were limited to 25 of the 40 patients (62.5\%). They received avastin (4\%), temozolomide (80\%), avastin + temozolomide (12\%), and irinotecan + avastin (4\%). Surgically, all patients received at least biopsy, and $21(52.5 \%)$ received total or subtotal resection. In the patients who entered treatment with a KPS score of 80 or better, their median KPS improved from an 80 to a 90 . For 


\section{Cureus}

those patients entering treatment with a KPS score of 70 or worse, they maintained a median KPS value of 70 before and after treatment. For the same group of patients that began treatment with a KPS of 80 or better, their median OS was 11.79 months as compared to 8.25 months for the group that began with 70 or worse $(\mathrm{P}=.071)$. OS broken down by KPS can be found in Figure $1 B$. Analysis showed that patients treated with GK, surgical resection, and chemotherapy (median OS of 14.2) had a significantly higher $(\mathrm{P}=.03)$ OS than patients treated with GK, chemotherapy, and no surgical resection (median OS of 8.9 months) and than patients with GK, surgical resection, and no chemotherapy (median OS of 5.37 months). OS for all patients was 10.9 months (Figure 1). OS by treatment modality can be found in Figure $1 C$.

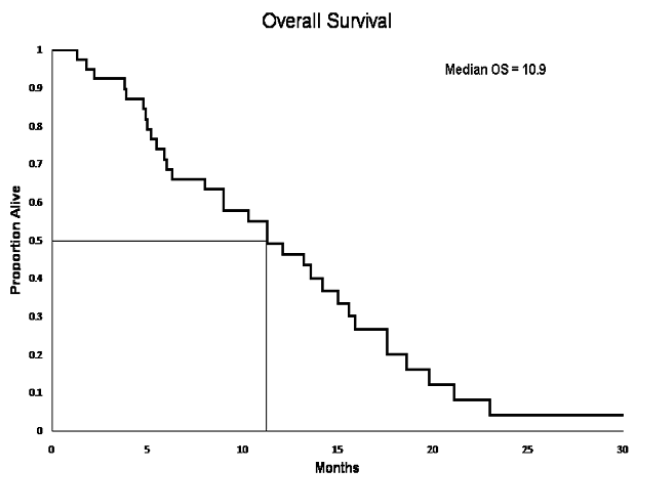

$1 \mathrm{~A}$

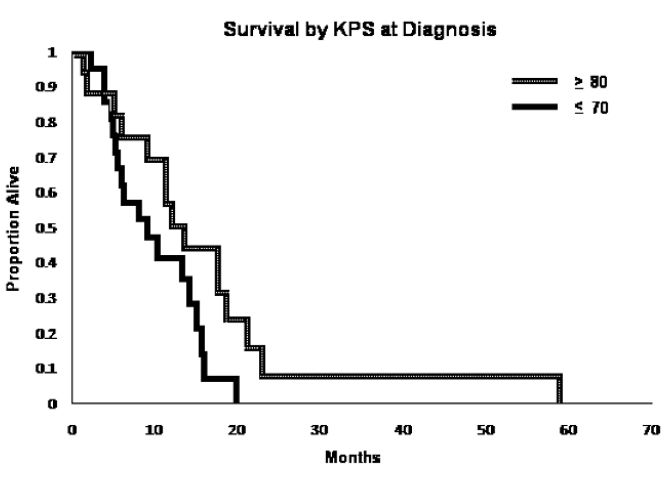

$1 B$

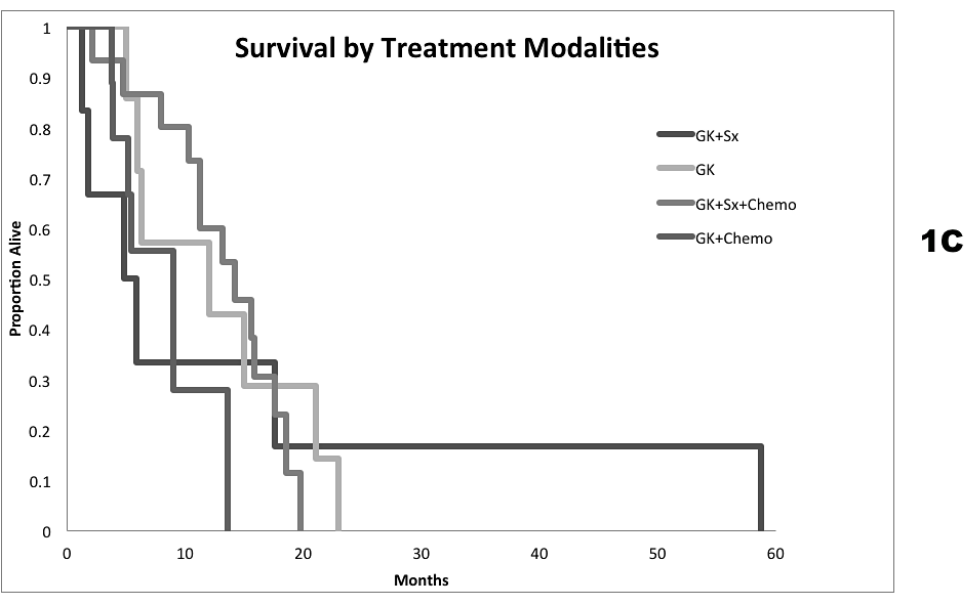

FIGURE 1: OS for all patients was 10.9 months 


\section{Cureus}

\section{Factor}

Age (years)

Mean \pm SD

Median

Range

Gender

Male

Female

KPS at diagnosis

$80 \leq$

$70 \geq$

Number of treatments

Number of treated lesions

Tumor location

Infratentorial

Supratentorial

Tumor volume

Mean

Median

Range

Bilateral disease

No

Yes

Overall Treatment
$\mathrm{N}(\%)$

$73.1 \pm 6.9$

75

65-95

$22(55)$

$18(45)$

$22(55)$

59

69

2

69

$.5-88$

39

1

TABLE 1: Patient and Treatment Characteristics 


\section{Cureus}

\section{Factor}

Overall

Treatment modality

GK,SX,Chemo

GK,SX

GK,Chemo

GK

KPS at diagnosis

$80 \leq$

$70 \geq$

8.25

\section{TABLE 2: Statistical analysis of survival diagnosis}

Abbreviations: Sx, surgery (greater than biopsy), GK, Gamma Knife ,Chemo, Chemotherapy , KPS, Karnofsky Performance Scale, GK, Gamma Knife

\section{Discussion}

The treatment of GBM in elderly populations has largely been left up to the discretion of physicians and treatment centers, due to the lack of a robust body of data and consensus. Different modalities have been employed in several combinations, ranging from conservative to aggressive. The standard of care currently is surgical resection combined with radiotherapy postoperatively. Mohan, et al. demonstrated that radiation was of value in increasing median OS [10]. Roa, et al. demonstrated that there was no statistical difference between standard radiation therapy (60 Gy in 30 fractions) or a shorter course (40Gy in 15 fractions) [11]. In studies on external beam radiation for GBM, Scott, et al. demonstrated that a combination of chemotherapy, surgical resection and fractionated RT in elderly patients was associated with a median OS of 13.3 months [12]. Our study results strongly support the use of Gamma Knife (GK) radiosurgery as an alternative to fractionated external beam radiation therapy.

The use of adjuvant chemotherapy as a standard of care for newly diagnosed patients with GBM was established by the study conducted by Stupp, et al. [13]. The addition of temozolomide to radiotherapy for newly diagnosed glioblastoma resulted in a clinically meaningful and statistically significant survival benefit with minimal additional toxicity. In elderly patients with GBM, the addition of chemotherapy to surgical and radiotherapy protocols was shown to be beneficial by Brandes, et al [6]. The trial showed that the addition of chemotherapy confers significant benefit to patients 65 or older. Our study results are in agreement with their conclusions. Although we did not parse the differences between different chemotherapeutic agents, our study showed that its addition increased OS for patients treated with GK and surgical resection (median of OS 14.3) from those who did not receive chemo (median OS of 5.37).

Randomized studies established the role of radiotherapy as part of the treatment of GBM in the 
late 1970's and 1980's [14-15]. This role has been confirmed in a more recently systematic review [16]. Building upon this evidence, dose escalation studies were performed but failed to show a benefit after radiosurgery in the setting of recurrent GBM was shown to be potentially promising. The RTOG 93-05 investigated the use of radiosurgery upfront in newly diagnosed GBM patients. This trial failed to show a benefit of this approach [9] and radiosurgery subsequently fell out of favor in this setting. However, we began utilizing Gamma Knife radiosurgery in place of conventional fractionated EBRT in elderly patients at upfront diagnosis to minimize the treatment time and effort required during the patient's remaining short life span. At that time median survival for elderly patients was only a handful of months. We agree with the literature that EBRT as part of aggressive treatment of GBM in elderly patients is warranted [12]. This alternative approach offers a truly hypofractionated schema using a single fraction regimen. This study confirms that our results are equivalent to those reported in the literature with EBRT (with short vs long course) for elderly patients with GBM [11].

A final consideration in the treatment of elderly patients with GBM is the cognitive implications of the RT modalities. Chang, et al. demonstrated that patients with brain metastasis treated with stereotactic radiosurgery (SRS), plus whole brain radiation therapy (WBRT), were at a greater risk of a significant decline in learning and memory function by four months compared with the group that received SRS alone. He goes on to recommend SRS and close clinical monitoring as the preferred treatment strategy to better preserve learning and memory in patients with newly diagnosed brain metastases [17]. Although the pathology is different, our study results are in agreement with Chang, et al. Of the patients that entered treatment with a KPS score of 80 or better, their median KPS improved from an 80 to a 90 . For those patients entering treatment with a KPS score of 70 or worse, they maintained a median KPS value of 70 before and after treatment. Additionally, our data supports Scott, et al. in that patients with an initial KPS of 80 or better had a median OS of 11.79 months as compared to those with initial KPS of 70 or below, median OS of 8.25 months. Our study supports the use of GK radiosurgery as a way to preserve performance status in elderly patients with GBM.

\section{Conclusions}

Aggressive treatment of the elderly is justified given the difference in the outcome of patients who receive Gamma Knife, surgery and chemotherapy versus no surgery and that addition of chemotherapy demonstrates increased OS. Furthermore, the results of patient cohort using radiosurgery alone are better than using conventional radiation therapy. If in fact further studies reveal that single treatment using radiosurgery is equivalent to five to six week fractionated paradigms, this saves the patients a significant amount of time spent in therapy, avoids toxicity, and allows for further therapy if necessary to distal sites with radiosurgery.

\section{Additional Information}

\section{Disclosures}

Human subjects: All authors have confirmed that this study did not involve human participants or tissue. Animal subjects: All authors have confirmed that this study did not involve animal subjects or tissue. Conflicts of interest: In compliance with the ICMJE uniform disclosure form, all authors declare the following: Payment/services info: All authors have declared that no financial support was received from any organization for the submitted work. Financial relationships: All authors have declared that they have no financial relationships at present or within the previous three years with any organizations that might have an interest in the submitted work. Other relationships: All authors have declared that there are no other relationships or activities that could appear to have influenced the submitted work.

\section{References}


1. Hess KR, Broglio KR, Bondy ML: Adult glioma incidence trends in the United States, 19772000. Cancer. 2000, 101:2293-2299. 10.1002/cncr.20621

2. Wrensch M, Minn Y, Chew T, Bondy M, Berger MS: Epidemiology of primary brain tumors: Current concepts and review of the literature. Neuro Oncol. 2002, 4:278-299. 10.1093/neuonc/4.4.278

3. Jukich PJ, McCarthy BJ, Surawicz TS, Freels S, Davis FG: Trends in incidence of primary brain tumors in the United States, 1985-1994. Neuro Oncol. 2001, 3:141-151.

10.1093/neuonc/3.3.141

4. Brandes AA, Compostella A, Blatt V, Tosoni A: Glioblastoma in the elderly: Current and future trends. Crit Rev Oncol Hematol. 2006, 60:256-266.

5. Brandes AA, Compostella A, Blatt V: Temozolomide concomitant and adjuvant to radiotherapy in elderly patients with glioblastoma: Correlation with MGMT promoter methylation status. Cancer. 2009, 115:3512-3518. 10.1002/cncr.24406

6. Brandes AA, Vastola F, Basso U, et al: A prospective study on glioblastoma in the elderly . Cancer. 2003, 97:657-662.

7. Combs SE, Wagner J, Bischof M, et al: Postoperative treatment of primary glioblastoma multiforme with radiation and concomitant temozolomide in elderly patients. Int J Radiat Oncol Biol Phys. 2008, 70:987-992. 10.1016/j.ijrobp.2007.07.2368

8. Vuorinen V, Hinkka S, Farkkila M, Jaaskelainen J: Debulking or biopsy of malignant glioma in elderly people-a randomized study. Acta Neurochir (Wien). 2003, 14:5-10.

9. Souhami,Luis, Zamorano, Lucia, Buckner, Jan, Riese, Nancy, Podgorsak, Ervin: RTOG 93-05: A Phase III Trial Comparing the use of radiosurgery follow be conventional radiotherapy with BCNU to conventional radiotherapy with BCNU for supratentorial glioblastoma multiforme. Radiation Therapy Oncology Group.. 1994.

10. Mohan DS, Suh JH, Phan JL, Kupelian PA, Cohen BH, Barnett GH: Outcome in elderly patients undergoing definitive surgery and radiation therapy for supratentorial glioblastoma multiforme at a tertiary care institution. Int J Radiat Oncol Biol Phys. 1998, 42:981-987.

11. Roa W, Brasher PM, Bauman G, et al: Abbreviated course of radiation therapy in older patients with glioblastoma multiforme: A prospective randomized clinical trial. J Clin Oncol. 2004, 22:1583-1588.

12. Scott JG, Suh JH, Elson P, Barnett GH, Vogelbaum MA, Peereboom DM, Stevens GH, Elinzano $\mathrm{H}$, Chao ST: Aggressive treatment is appropriate for glioblastoma multiforme patients 70 years old or older: a retrospective review of 206 cases. Neuro Oncol. 2011, 13:428-436. 10.1093/neuonc/nor005

13. Stupp R, Mason WP, van den Bent MJ, et al.: Radiotherapy plus Concomitant and Adjuvant Temozolomide for Glioblastoma. N Engl J Med. 2005, 352:987-996.

14. Walker MD, Alexander E Jr, Hunt WE: Evaluation of BCNU and/or radiotherapy in the treatment of anaplastic gliomas. A cooperative clinical trial.. J Neurosurg. 1978, 49:333-343.

15. Walker MD, Green SB, Byar DP, et al: Randomized comparisons of radiotherapy and nitrosoureas for the treatment of malignant glioma after surgery. N Engl J Med. 1980, 303:1323-1329.

16. Laperriere N, Zuraw L, Cairncross G, Cancer Care Ontario Practice Guidelines Initiative Neuro-Oncology Disease Site Group: Radiotherapy for newly diagnosied malignant glioma in adults: A systematic review. Radiother Oncol. 2002, 64:259-273.

17. Chang EL, Wefel JS, Hess KR, Allen PK, Lang FF, Kornguth DG, Arbuckle RB, Swint JM, Shiu AS, Maor MH, Meyers CA: Neurocognition in patients with brain metastases treated with radiosurgery or radiosurgery plus whole-brain irradiation: a randomised controlled trial. Lancet Oncol. 2009, 10:1037-1044. 10.1016/S1470-2045(09)70263-3 